\title{
Perflutren Lipid Microspheres
}

National Cancer Institute

\section{Source}

National Cancer Institute. Perflutren Lipid Microspheres. NCI Thesaurus. Code C74071.

An injectable suspension of liposome-encapsuled microspheres containing the

fluorocarbon gas perflutren for contrast enhancement in ultrasound procedures.

Because the acoustic impedance of perflutren lipid microspheres is much lower than that of blood, impinging ultrasound waves are scattered and reflected at the microsphereblood interface and may be visualized with ultrasound imaging. 